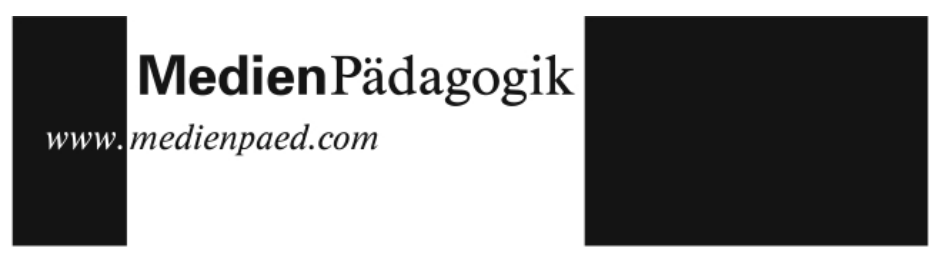

Rezensionen

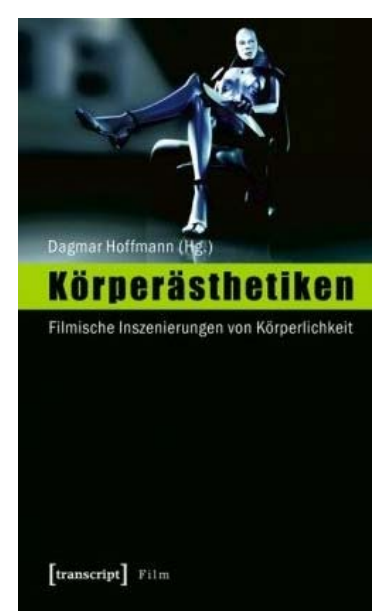

\section{Körper im Film}

Sammelbände zu körpersoziologischen Fragestellungen erfreuen sich seit einigen Jahren größerer Beliebtheit. Neu ist hingegen die Auseinandersetzung mit Körpern im Film. Diese Lücke füllt nun der bei Transcript erschienene und von Dagmar Hoffmann herausgegebene Sammelband Körperästhetiken. Filmische Inszenierung von Körperlichkeit.

Der Band zeigt die Darstellung und die symbolische Aufladung von Körpern in Kinofilmen am Beispiel von kämpfenden Menschmaschinen in Terminator II, Heldenkörpern in der Comicverfilmung 300, Soldatenkörpern in aktuellen Hollywood-Kriegsfilmen, virtuellen und fremdgesteuerten Körpern im Film Matrix, sportgestählten Körpern im Boxdrama Million Dollar Baby, adipösen Körpern in Gilbert Grape - Irgendwo in lowa, gemaßregelten Körpern in der Psychiatrie im Film Girl - Interrupted, transgeschlechtlichen Körpern im Roadmovie Transamerica, alternden Körpern in There will be blood, Körperdarstellungen im Spiegel der Filmmusik zu Stanley Kubricks Eyes Wide Shut, Frauenkörpern im Vergleich von Eyes Wide Shut und Arthur Schnitzlers Traumnovelle, postkoloniale Körperinszenierungen in der Bollywood-Romanze Dilwale dulhania le jayenge, Körperbildern bzw. körperlosen Bildern in den pseudodokumentarischen Spielfilmen The Blairwitch Project und Cloverfield, sowie die Bedeutung von Erotik und Nacktheit im DDR-Film.

Dagmar Hoffmann eröffnet den Band mit einer Bestandsaufnahme von Körperbildern und Rollenklischees in den beliebtesten Filmen von Casablanca bis Pulp Fiction. Die erfolgreichsten Produktionen waren überwiegend 
Kriegs-, Gangster, Spionage, und Mafiafilme mit fast immer stereotypen männlichen Hauptrollen und nicht minder schablonenhaften weiblichen Nebenrollen, so Hoffmann. Unter den derzeit bestbezahlten Hollywood-Darsteller/innen stellt die Herausgeberin eine geringe Variabilität hinsichtlich Alter, Hautfarbe, Körperbau, und -größe fest; wobei diese bei Männern größer sei als bei Frauen. Dicke Körper landeten fast immer im komischen Fach. Erotische oder ernste Rollen seien hingegen schlanken Darsteller/innen vorbehalten. Die Mehrzahl der Hollywood-Beaus sei in Sachen Schönheit und Anmutung für ihre Zuschauer/innen unerreichbar. Gleichzeitig stelle das gängige Schönheitsideal aber so etwas wie den kleinsten gemeinsamen Nenner des Publikums dar. Leider fehlt der thematischen Einführung von Hoffmann die üblicherweise Sammelbänden vorangestellte Kurzvorstellung und inhaltliche Einordnung der Beiträge.

Abgesehen von zwei Aufsätzen zu Produktionen aus Babelsberg und Bombay beziehen sich alle Beiträge auf Hollywoodfilme der letzten zwanzig Jahre. Einige von ihnen sollen hier näher vorgestellt werden.

Robert Gugutzer zeigt, wie in Million Dollar Baby Körper stereotyp und vorurteilsbehaftet dargestellt werden. Das mit vier Oscars ausgezeichnete Sportmelodram von und mit Clint Eastwood erzählt die Geschichte einer Kellnerin aus einfachen Verhältnissen, die es trotz fortgeschrittenen Alters durch eiserne Disziplin und die väterliche Anleitung ihres Trainers bis ins Finale der Boxweltmeisterschaft bringt. Erst dort wird sie von ihrer Konkurrentin, dargestellt von einer afroamerikanischen Schauspielerin, mit unfairen Mitteln gestoppt und schwer verletzt. «Der Kontrast zwischen den Körperbildern einer weißen und einer schwarzen Boxerin könnte schematischer kaum sein. Von der Farbsymbolik der Kleidung über die Frisuren und Blicke bis hin zu Körperhaltung und -bewegung vermitteln die Körperbilder eine eindeutige Botschaft» (131f.), analysiert Gugutzer den rassistischen Subtext des Films.

In Gilbert Grape - Irgendwo in lowa wird die Geschichte einer Frau erzählt, die nach dem Freitod ihres Mannes massiv zunimmt und durch ihre Unbeweglichkeit und Hilfsbedürftigkeit ihre Kinder gewissermaßen in Geiselhaft nimmt. Die Erlösung für die Familie scheint allein im Tod der nicht nur körperlich monströsen, sondern auch sonst als schrill, laut und ungezügelt dar- 
gestellten Mutter zu bestehen. Trotz aller Kritik an der klischeehaften und überzogenen Darstellung der adipösen Mutter sei der Film mehr als nur die Projektion von einfachen Phantasien und Ängsten auf einer Leinwand, resümiert Sandra Günter. «Es ist ein Film der 1990er, der die diskursive Konstruktion des Körpers durch zeit- und kulturspezifische Wissensformen, Denk- und Deutungsmuster veranschaulicht. Es ist ein vielschichtiges, zeitund biopolitisches Dokument» (160), schließt sie mit Bezug auf Foucaults Gouvernementalitätstheorie.

Als Entdramatisierung der Geschlechterrollen beschreiben Heike Klippel und Thomas Knieper die Geschichte das Zusammentreffen des als Frau lebenden Vaters mit seinem Sohn im Roadmovie Transamerica. Der Wunsch des biologischen Mannes, dargestellt durch die Schauspielerin Felicity Huffman, nach einer Geschlechtsumwandlung, wird im Film als völlig legitim und natürlich beschrieben. Durch seine empathische und unaufgeregte Erzählweise trage Transamerica dazu bei, die gesellschaftliche Konstruktion von Zweigeschlechtlichkeit in Frage zu stellen, resümieren Klippel und Knieper. Erst seit wenigen Jahren werden Filme aus Indien von einem Massenpublikum in Europa wahrgenommen. Seit einiger Zeit werden bekannte Bollywoodproduktionen auch im deutschen Fernsehen ausgestrahlt. Florian Krauss analysiert eine der erfolgreichsten Bollywood-Produktionen der vergangenen Jahre und kommt dabei zu spannenden Erkenntnissen. Nicht nur in Sachen rassistischer Stereotype gibt es Gemeinsamkeiten zwischen Bollywood und Hollywood: wenn etwa dunkelhäutige Schauspieler aufs komische Fach beschränkt bleiben und die Frauen in den Hauptrollen auffallend hellhäutig und schlank sind. Auch die Inszenierung exotischer Kulissen folgt eurozentristischen Mustern, wenn auch unter umgekehrten Vorzeichen. «Wir sehen non resindent Indians im Interrail-Urlaub, aber nahezu keine weißen Europäer. Eine Perspektive, die sich kaum für den «Anderen〉 interessiert und das eroberte Land allein als pittoresk-exotische Kulisse begreift, erfährt ihre Umkehrung.» (318). 


\section{Rezensionen}

Einen Exkurs stellt der letzte Beitrag des Sammelbandes von Dieter Wiedemann zu Nacktheit und Erotik im DDR-Kino dar. Der Titel ist irreführend: behandelt der Beitrag doch eher die Frage, inwieweit sich die Filme gegen die miefige Sexualmoral der DDR auflehnten. Nacktheit und explizite Erotik spielten dabei eine viel geringere Rolle als die Darstellung außerehelicher Affären und anderer unkonventioneller Liebesbeziehungen.

Ingesamt bietet der Band Körperästhetiken viele interessante Überlegungen zur Darstellung von Körper in zeitgenössischen Filmen und den durch sie vermittelten Subtext. Neben den lesenswerten Einzelbeispielen wären allerdings mehr Übersichtsartikel wünschenswert gewesen, die Trends und Entwicklungen genre- und zeitübergreifend darstellen und in größere politischökonomische Zusammenhänge einordnen.

Friedrich Schorb 